\title{
High-quality Motion Deblurring from a Single Image *
}

\author{
Qi Shan \\ Jiaya Jia \\ Department of Computer Science and Engineering \\ The Chinese University of Hong Kong
}

\author{
Aseem Agarwala \\ Adobe Systems, Inc.
}
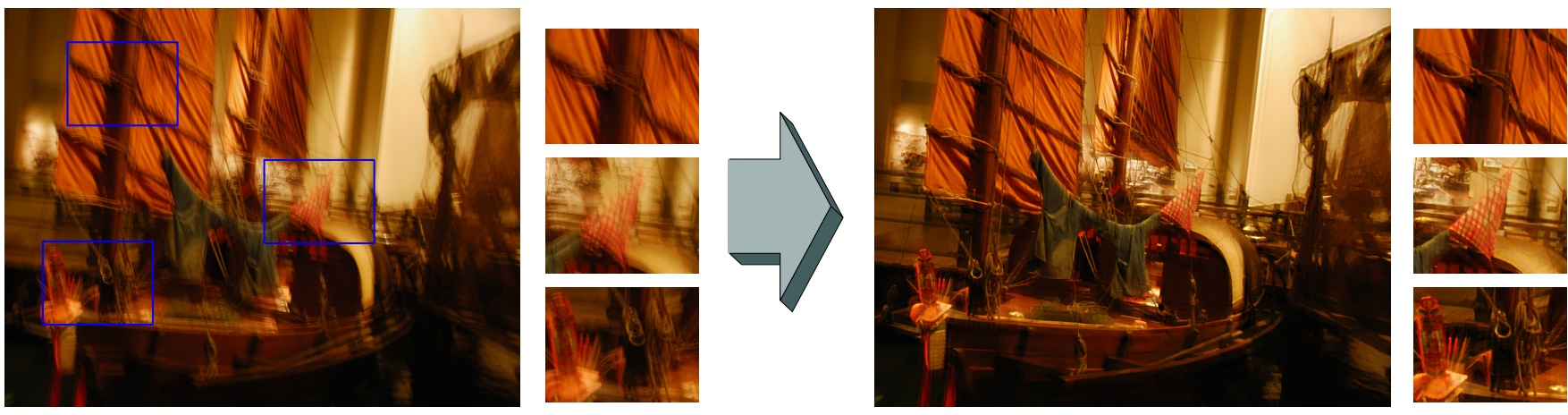

Figure 1 High quality single image motion-deblurring. The left sub-figure shows one captured image using a hand-held camera under dim light. It is severely blurred by an unknown kernel. The right sub-figure shows our deblurred image result computed by estimating both the blur kernel and the unblurred latent image. We show several close-ups of blurred/unblurred image regions for comparison.

\begin{abstract}
We present a new algorithm for removing motion blur from a single image. Our method computes a deblurred image using a unified probabilistic model of both blur kernel estimation and unblurred image restoration. We present an analysis of the causes of common artifacts found in current deblurring methods, and then introduce several novel terms within this probabilistic model that are inspired by our analysis. These terms include a model of the spatial randomness of noise in the blurred image, as well a new local smoothness prior that reduces ringing artifacts by constraining contrast in the unblurred image wherever the blurred image exhibits low contrast. Finally, we describe an efficient optimization scheme that alternates between blur kernel estimation and unblurred image restoration until convergence. As a result of these steps, we are able to produce high quality deblurred results in low computation time. We are even able to produce results of comparable quality to techniques that require additional input images beyond a single blurry photograph, and to methods that require additional hardware.
\end{abstract}

Keywords: motion deblurring, ringing artifacts, image enhancement, filtering

\section{Introduction}

One of the most common artifacts in digital photography is motion blur caused by camera shake. In many situations there simply is not

${ }^{*}$ http://www.cse.cuhk.edu.hk/\% 7eleojia/projects/motion\%5fdeblurring/

ACM Reference Format

Shan, Q., Jia, J., Agarwala, A. 2008. High-quality Motion Deblurring from a Single Image. ACM Trans. Graph. 27, 3, Article 73 (August 2008), 10 pages. DOI = 10.1145/1360612.1360672 http://doi.acm. org/10.1145/1360612.1360672.

\section{Copyright Notice}

Permission to make digital or hard copies of part or all of this work for personal or classroom use is granted without fee provided that copies are not made or distributed for profit or direct commercial advantage and that copies show this notice on the first page or initial screen of a display along with the full citation. Copyrights for components of this work owned by others than ACM must be honored. Abstracting with credit is permitted. To copy otherwise, to republish, to post on servers, to redistribute to lists, or to use any component of this work in other works requires prior specific permission and/or a fee. Permissions may be requested from Publications Dept., ACM, Inc., 2 Penn Plaza, Suite 701, New York, NY 10121-0701, fax + (212) 869-0481, or permissions@acm.org.

(ㅇ) 2008 ACM 0730-0301/2008/03-ART73 \$5.00 DOI 10.1145/1360612.1360672

http://doi.acm.org/10.1145/1360612.1360672 enough light to avoid using a long shutter speed, and the inevitable result is that many of our snapshots come out blurry and disappointing. Recovering an un-blurred image from a single, motion-blurred photograph has long been a fundamental research problem in digital imaging. If one assumes that the blur kernel - or point spread function (PSF) - is shift-invariant, the problem reduces to that of image deconvolution. Image deconvolution can be further separated into the blind and non-blind cases. In non-blind deconvolution, the motion blur kernel is assumed to be known or computed elsewhere; the only task remaining is to estimate the unblurred latent image. Traditional methods such as Weiner filtering [Wiener 1964] and Richardson-Lucy (RL) deconvolution [Lucy 1974] were proposed decades ago, but are still widely used in many image restoration tasks nowadays because they are simple and efficient. However, these methods tend to suffer from unpleasant ringing artifacts that appear near strong edges. In the case of blind deconvolution [Fergus et al. 2006; Jia 2007], the problem is even more ill-posed, since both the blur kernel and latent image are assumed unknown. The complexity of natural image structures and diversity of blur kernel shapes make it easy to over- or under-fit probabilistic priors [Fergus et al. 2006].

In this paper, we begin our investigation of the blind deconvolution problem by exploring the major causes of visual artifacts such as ringing. Our study shows that current deconvolution methods can perform sufficiently well if both the blurry image contains no noise and the blur kernel contains no error. We therefore observe that a better model of inherent image noise and a more explicit handling of visual artifacts caused by blur kernel estimate errors should substantially improve results. Based on these ideas, we propose a unified probabilistic model of both blind and non-blind deconvolution and solve the corresponding Maximum a Posteriori (MAP) problem by an advanced iterative optimization that alternates between blur kernel refinement and image restoration until convergence. Our algorithm can be initialized with a rough kernel estimate (e.g., a straight line), and our optimization is able to converge to a result that preserves complex image structures and fine edge details, while avoiding ringing artifacts, as shown in Figure 1.

To accomplish these results, our technique benefits from three main contributions. The first is a new model of the spatially random dis- 
tribution of image noise. This model helps us to separate the errors that arise during image noise estimation and blur kernel estimation, the mixing of which is a key source of artifacts in previous methods. Our second contribution is a new smoothness constraint that we impose on the latent image in areas where the observed image has low contrast. This constraint is very effective in suppressing ringing artifacts not only in smooth areas but also in nearby textured ones. The effects of this constraint propagate to the kernel refinement stage, as well. Our final contribution is an efficient optimization algorithm employing advanced optimization schemes and techniques, such as variable substitutions and Plancherel's theorem, that allow computationally intensive optimization steps to be performed in the frequency domain.

We demonstrate our method by presenting its results and comparisons to the output of several state-of-the-art single image deblurring methods. We also compare our results to those produced by algorithms that require additional input beyond a single image (e.g., multiple images) and an algorithm that utilizes specialized hardware; surprisingly, most of our results are comparable even though only a single image is used as input.

\section{Related Work}

We first review techniques for non-blind deconvolution, where the blur kernel is known and only a latent image must be recovered from the observed, blurry image. The most common technique is Richardson-Lucy (RL) deconvolution [1974], which computes the latent image with the assumption that its pixel intensities conform to a Poisson distribution. Donatelli et al. [2006] use a PDE-based model to recover a latent image with reduced ringing by incorporating an anti-reflective boundary condition and a re-blurring step. Several approaches proposed in the signal processing community solve the deconvolution problem in the wavelet domain or the frequency domain [Neelamani et al. 2004]; many of these papers lack experiments in de-blurring real photographs, and few of them attempt to model error in the estimated kernel. Levin et al. [2007] use a sparse derivative prior to avoid ringing artifacts in deconvolution. Most non-blind deconvolution methods assume that the blur kernel contains no errors, however, and as we show later with a comparison, even small kernel errors or image noise can lead to significant artifacts. Finally, many of these deconvolution methods require complex parameter settings and long computation times.

Blind deconvolution is a significantly more challenging and illposed problem, since the blur kernel is also unknown. Some techniques make the problem more tractable by leveraging additional input, such as multiple images. Rav-Acha et al. [2005] leverage the information in two motion blurred images, while Yuan et al. [2007] use a pair of images, one blurry and one noisy, to facilitate capture in low light conditions. Other motion deblurring systems take advantage of additional, specialized hardware. Ben-Ezra and Nayar [2004] attach a low-resolution video camera to a high-resolution still camera to help in recording the blur kernel. Raskar et al. [2006] flutter the opening and closing of the camera shutter during exposure to minimize the loss of high spatial frequencies. In their method, the object motion path must be specified by the user. In contrast to all these methods, our technique operates on a single image and requires no additional hardware.

The most ill-posed problem is single-image blind deconvolution, which must both estimate the PSF and latent image. Early approaches usually assume simple parametric models for the PSF such as a low-pass filter in the frequency domain [Kim and Paik 1998] or a sum of normal distributions [Likas and Galatsanos 2004]. Fergus et al. [2006] showed that blur kernels are often complex and sharp; they use ensemble learning [Miskin and MacKay 2000] to recover a blur kernel while assuming a certain

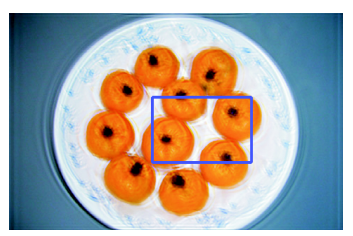

(a)

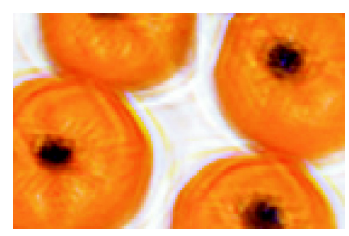

(b)
Figure 2 Ringing artifacts in image deconvolution. (a) A blind deconvolution result. (b) A magnified patch from (a). Ringing artifacts are visible around strong edges.

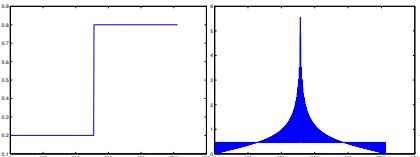

(a) (b)

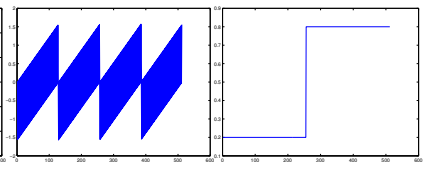

(c) (d)
Figure 3 A step signal constructed by finite Fourier basis functions. (a) The ground truth step signal. (b) The magnitude of the coefficients of the Fourier basis functions. (c) The phase of the Fourier basis coefficients. (d) The reconstructed signal from the 512 Fourier basis functions, with PSNR of 319.40. The loss is far below what humans can perceive.

statistical distribution for natural image gradients. A variational method is used to approximate the posterior distribution, and then Richardson-Lucy is used for deconvolution. Jia [2007] recovered a PSF from the perspective of transparency by assuming the transparency map of a clear foreground object should be two-tone. This method is limited by a need to find regions that produce highquality matting results. A significant difference between our approach and previous work is that we create a unified probabilistic framework for both blur kernel estimation and latent image recovery; by allowing these two estimation problems to interact we can better avoid local minima and ringing artifacts.

\section{Analysis of Ringing Artifacts}

We begin with an analysis of sources of error in blind and non-blind deconvolution. Shift-invariant motion blur is commonly modeled as a convolution process

$$
\mathbf{I}=\mathbf{L} \otimes \mathbf{f}+\mathbf{n}
$$

where $\mathbf{I}, \mathbf{L}$, and $\mathbf{n}$ represent the degraded image, latent unblurred image, and additive noise, respectively. $\otimes$ denotes the convolution operator, and $\mathbf{f}$ is a linear shift-invariant point spread function (PSF).

One of the major problems in latent image restoration is the presence of ringing artifacts, such as those shown in Figure 2. Ringing artifacts are dark and light ripples that appear near strong edges after deconvolution. It is commonly believed [Yuan et al. 2007] that ringing artifacts are Gibbs phenomena from an inability of finite Fourier basis functions to model the kind of step signals that are commonly found in natural images. However, we have found that a reasonable number of finite Fourier basis functions can reconstruct natural image structures with an imperceivable amount of loss. To illustrate, in Figure 3 we show that a 1D step signal can be reconstructed by 512 finite Fourier basis functions; we have found similar accuracy in discrete image space, and using hundreds of Fourier basis functions is not computationally expensive at all.

If deconvolution artifacts are not caused by Gibbs phenomenon, what are they caused by? In our experiments, we find that both inaccurately modeled image noise $\mathbf{n}$ and errors in the estimated PSF f are the main cause of ringing artifacts. To illustrate, we show several examples in Figure 4. In the first two rows we show two 1D 


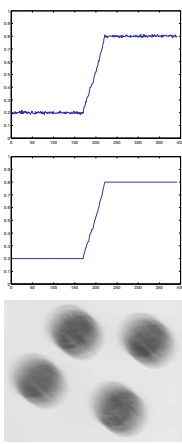

(a) $I$

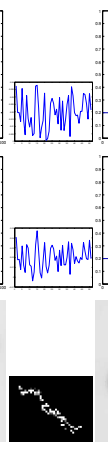

(b) $f$
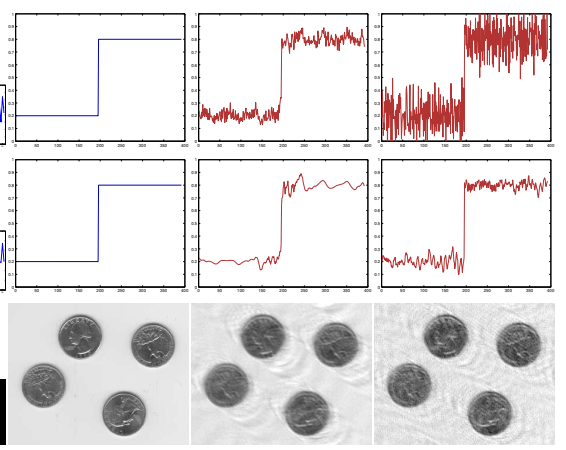

(c) $L$
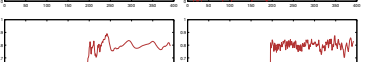

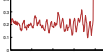

Figure 4 Noise in deconvolution. The first two rows show 1D signal examples corrupted with signal noise and kernel noise, respectively. The last row shows an image example in the presence of both image noise and kernel noise. For all rows, (a) shows the observed signals. (b) The kernel. (c) The ground truth signal. (d) The reconstructed signal using RL method. (e) The reconstructed signal by Wiener filtering. Artifact can be seen in the deconvolution results.

signals where the observed signal and blur kernel are contaminated by a small amount of noise, respectively, causing the RL method and Wiener filtering to produce unsatisfactory results. In the third row we show a $2 \mathrm{D}$ image where both the blurred image and the blur kernel are corrupted with noise. Although the results from the RL method and Winer filtering preserve strong edges, perceivable artifacts are also introduced.

To analyze the problems cause by image noise and kernel error, let us model the kernel and latent image as the sum of their current estimates $\mathbf{f}^{\prime}$ and $\mathbf{L}^{\prime}$ and the errors $\Delta \mathbf{f}$ and $\Delta \mathbf{L}$ :

$$
\begin{aligned}
\mathbf{I} & =\left(\mathbf{L}^{\prime}+\Delta \mathbf{L}\right) \otimes\left(\mathbf{f}^{\prime}+\Delta \mathbf{f}\right)+\mathbf{n} \\
& =\mathbf{L}^{\prime} \otimes \mathbf{f}^{\prime}+\Delta \mathbf{L} \otimes \mathbf{f}^{\prime}+\mathbf{L}^{\prime} \otimes \Delta \mathbf{f}+\Delta \mathbf{L} \otimes \Delta \mathbf{f}+\mathbf{n} .
\end{aligned}
$$

In the above equation, we can see that if observed noise $\mathbf{n}$ is not modeled well, it is easy for an estimation algorithm to mistake errors $\Delta \mathbf{f}$ and $\Delta \mathbf{L}$ as part of the noise $\mathbf{n}$, making the estimation process unstable and challenging to solve. Previous techniques typically model image noise $\mathbf{n}$ or its gradient $\partial \mathbf{n}$ as following zeromean Gaussian distributions. This model is weak and subject to the risk outlined above because it does not capture an important property of image noise, which is that image noise exhibits spatial randomness. To illustrate, in Figure 6(d) we show that replacing our stronger model of noise (described in the next section) with a simple zero-mean Gaussian yields a noise estimate $(\mathbf{L} \otimes \mathbf{f}-\mathbf{I})$ that is clearly structured and not spatially random.

To summarize this section, we conclude that deconvolution ringing artifacts are not caused by Gibbs phenomenon, but rather primarily by observed image noise and kernel estimate errors that mix during estimation, resulting in estimated image noise that exhibits spatial structure. In the next section, we propose a unified probabilistic model to address this issue and suppress visual artifacts.

\section{Our model}

Our probabilistic model unifies blind and non-blind deconvolutions into a single MAP formulation. Our algorithm for motion deblurring then iterates between updating the blur kernel and estimating the latent image, as shown in Figure 5. By Bayes' theorem,

$$
p(\mathbf{L}, \mathbf{f} \mid \mathbf{I}) \propto p(\mathbf{I} \mid \mathbf{L}, \mathbf{f}) p(\mathbf{L}) p(\mathbf{f})
$$

where $p(\mathbf{I} \mid \mathbf{L}, \mathbf{f})$ represents the likelihood and $p(\mathbf{L})$ and $p(\mathbf{f})$ denote the priors on the latent image and the blur kernel. We now define

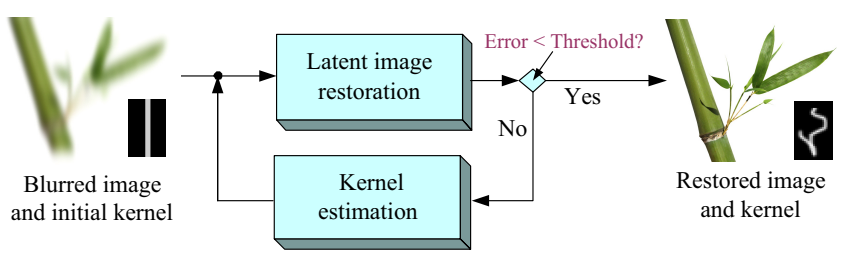

Figure 5 Overview of our algorithm. Given an observed blurred image and an initial kernel, our proposed method iteratively estimates the latent image and refines the blur kernel until convergence.

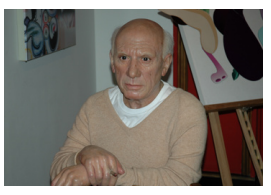

(a)

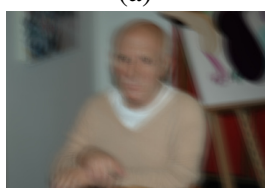

(b)

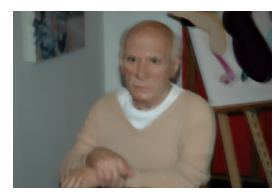

(c)

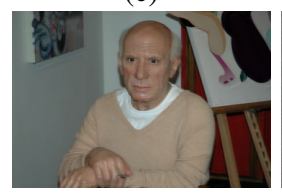

(e)

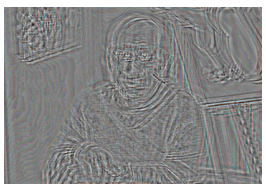

(d)

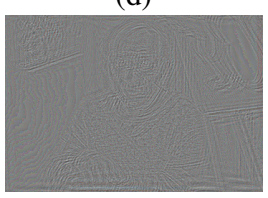

(f)
Figure 6 Effect of our likelihood model. (a) The ground truth latent image. (b) The blurred image $\mathbf{I}$. (c) The latent image $\mathbf{L}^{a}$ computed by our algorithm using the simple likelihood definition $\prod_{i} N\left(n_{i} \mid 0, \zeta_{0}\right)$. (d) The computed image noise map $\mathbf{n}$ from (c) by $\mathbf{n}=\mathbf{L}^{a} \otimes \mathbf{f}^{a}-\mathbf{I}$. (e) The latent image result $\mathbf{L}^{b}$ recovered by using the complete likelihood definition (3). (f) The computed noise map from (e) by $\mathbf{n}=\mathbf{L}^{b} \otimes \mathbf{f}^{b}-$ I. $\mathbf{f}^{a}$ and $\mathbf{f}^{b}$ are estimated kernels, and (d) and (f) are normalized for visualization.

these terms, and describe our optimization in the next section.

\subsection{Definition of the probability terms}

Likelihood $p(\mathbf{I} \mid \mathbf{L}, \mathbf{f})$. The likelihood of an observed image given the latent image and blur kernel is based on the convolution model $\mathbf{n}=\mathbf{L} \otimes \mathbf{f}-\mathbf{I}$. Image noise $\mathbf{n}$ is modeled as a set of independent and identically distributed (i.i.d.) noise random variables for all pixels, each of which follows a Gaussian distribution.

In previous work, the likelihood term is simply written as $\prod_{i} N\left(n_{i} \mid 0, \zeta_{0}\right)$ [Jia 2007] or $\prod_{i} N\left(\nabla n_{i} \mid 0, \zeta_{1}\right)$ [Fergus et al. 2006], which considers the noise for all pixel $i$ 's, where $\zeta_{0}$ and $\zeta_{1}$ are the standard deviations (SDs). However, as we discussed in the previous section, these models do not capture at all the spatial randomness that we would expect in noise.

In our formulation, we model the spatial randomness of noise by constraining several orders of its derivatives. In the rest of this paper, the image coordinate $(x, y)$ and pixel $i$ are alternatively used. Similarly, we sometimes represent $n_{i}$ by $n(x, y)$ to facilitate the representation of partial derivatives.

We denote the partial derivatives of $\mathbf{n}$ in the two directions as $\partial_{x} \mathbf{n}$ and $\partial_{y} \mathbf{n}$ respectively. They can be computed by the forward difference between variables representing neighboring pixels, i.e., $\partial_{x} n(x, y)=n(x+1, y)-n(x, y)$ and $\partial_{y} n(x, y)=$ $n(x, y+1)-n(x, y)$. Since each $n$ is a i.i.d. random variable following an independent Gaussian distribution with standard deviation $\zeta_{0}$, it is proven in [Simon 2002] that $\partial_{x} n$ and $\partial_{y} n$ also follow i.i.d. Gaussian distributions $N\left(\partial_{x} n_{i} \mid 0, \zeta_{1}\right)$ and $N\left(\partial_{y} n_{i} \mid 0, \zeta_{1}\right)$ with standard deviation (SD) $\zeta_{1}=\sqrt{2} \zeta_{0}$.

In regard to higher-order partial derivatives of image noise, it can be 
easily shown that the i.i.d. property also makes them follow Gaussian distributions with different standard deviations. We recursively define the high-order partial derivatives of image noise using forward differences. For example, $\partial_{x x}$ is calculated by

$$
\begin{aligned}
& \partial_{x x} n(x, y)=\partial_{x} n(x+1, y)-\partial_{x} n(x, y) \\
& \quad=(n(x+2, y)-n(x+1, y))-(n(x+1, y)-n(x, y)) .
\end{aligned}
$$

Since $\partial_{x} n(x, y+1)$ and $\partial_{x} n(x, y)$ are both i.i.d. random variables, the value of $\partial_{x x} n(x, y)$ can also be proven [Simon 2002] to follow a Gaussian distribution with $\operatorname{SD} \zeta_{2}=\sqrt{2} \cdot \zeta_{1}=\sqrt{2^{2}} \zeta_{0}$.

For simplicity's sake, in the following formulation, we use $\partial^{*}$ to represent the operator of any partial derivative and $\kappa\left(\partial^{*}\right)$ to represent its order. For example, $\partial^{*}=\partial_{x y}$ and $\kappa\left(\partial^{*}\right)=2$. For any $\partial^{*}$ with $\kappa\left(\partial^{*}\right)=q$, where $q>1, \partial^{*} n_{i}$ follows an independent Gaussian distribution with $\operatorname{SD} \zeta_{q}=\sqrt{2} \cdot \zeta_{q-1}=\sqrt{2^{q}} \zeta_{0}$.

To model the image noise as spatially i.i.d., we combine the constraints signifying that $\partial^{*} \mathbf{n}$ with different orders all follow Gaussian distributions, and define the likelihood as

$$
\begin{aligned}
p(\mathbf{I} \mid \mathbf{L}, \mathbf{f}) & =\prod_{\partial^{*} \in \Theta} \prod_{i} N\left(\partial^{*} n_{i} \mid 0, \zeta_{\kappa\left(\partial^{*}\right)}\right) \\
& =\prod_{\partial^{*} \in \Theta} \prod_{i} N\left(\partial^{*} I_{i} \mid \partial^{*} I_{i}^{c}, \zeta_{\kappa\left(\partial^{*}\right)}\right),
\end{aligned}
$$

where $I_{i} \in \mathbf{I}$, denotes the pixel value, $I_{i}^{c}$ is the pixel with coordinate $i$ in the reconvolved image $\mathbf{I}^{c}=\mathbf{L} \otimes \mathbf{f}$, and $\Theta$ represents a set consisting of all the partial derivative operators that we use (we set $\Theta=\left\{\partial^{0}, \partial_{x}, \partial_{y}, \partial_{x x}, \partial_{x y}, \partial_{y y}\right\}$ and define $\left.\partial^{0} n_{i}=n_{i}\right)$. We compute derivatives with a maximum order of two because our experiments show that these derivatives are sufficient to produce good results. Note that using the likelihood defined in (3) does not increase computational difficulty compared to only using $\prod_{i} N\left(n_{i} \mid 0, \zeta_{0}\right)$ in our optimization.

Figure 6(e) shows that our likelihood model (3) can significantly improve the quality of the image restoration result. The computed noise map in (f) by $\mathbf{n}=\mathbf{L} \otimes \mathbf{f}-\mathbf{I}$ contains less image structure compare to map (d), which was computed without modeling the noise derivatives in different orders.

Prior $p(\mathbf{f})$. In the kernel estimation stage, it is commonly observed that since a motion kernel identifies the path of the camera it tends to be sparse, with most values close to zero. We therefore model the values in a blur kernel as exponentially distributed

$$
p(\mathbf{f})=\prod_{j} e^{-\tau f_{j}}, \quad f_{j} \geq 0,
$$

where $\tau$ is the rate parameter and $j$ indexes over elements in the blur kernel.

Prior $p(\mathbf{L})$. We design the latent image prior $p(\mathbf{L})$ to satisfy two objectives. One one hand, the prior should serve as a regularization term that reduces the ill-posedness of the deconvolution problem. On the other hand, the prior should help to reduce ringing artifacts during latent image restoration. We thus introduce two components for $p(\mathbf{L})$ : the global prior $p_{g}(\mathbf{L})$ and the local prior $p_{l}(\mathbf{L})$, i.e.,

$$
p(\mathbf{L})=p_{g}(\mathbf{L}) p_{l}(\mathbf{L}) .
$$

Global prior $p_{g}(\mathbf{L})$. Recent research in natural image statistics shows that natural image gradients follow a heavy-tailed distribution [Roth and Black 2005; Weiss and Freeman 2007] which can be learnt from sample images. Such a distribution provides a natural prior for the statistics of the latent image.

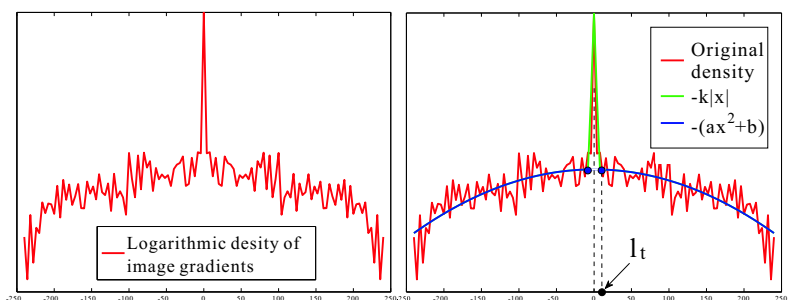

(a)

(b)

Figure 7 (a) The curve of the logarithmic density of image gradients. It is computed using information collected from 10 natural images. (b) We construct function $\Phi(x)$ to approximate the logarithmic density, as shown in green and blue.

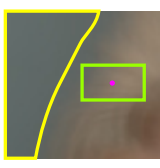

(a)

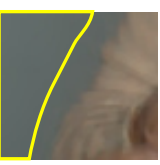

(b)

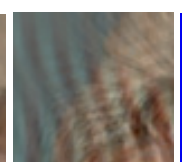

(c)

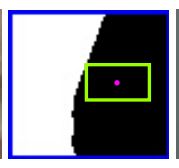

(d)

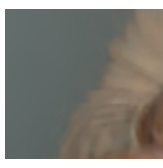

(e)
Figure 8 Local prior demonstration. (a) A blurred image patch extracted from Figure 9(b). The yellow curve encloses a smooth region. (b) The corresponding unblurred image patch. (c) The restoration result by our method without incorporating $p_{l}$. The ringing artifact is propagated even to the originally smooth region. (d) We compute $\Omega$ containing all pixels shown in white to suppress ringing. (e) Our restoration result incorporating $p_{l}$, the ringing artifact is suppressed not only in smooth region but also in textured one.

To illustrate, in Figure 7 we show the logarithmic image gradient distribution histogram from 10 natural images. In [Fergus et al. 2006], a mixture of $K$ Gaussian distributions are used to approximate the gradient distribution: $\prod_{i} \sum_{k=1}^{K} \omega_{k} N\left(\partial L_{i} \mid 0, \varsigma_{k}\right)$, where $i$ indexes over image pixels, $\omega_{k}$ and $\varsigma_{k}$ denote the weight and the standard deviation of the $k$ 'th Gaussian distribution. However, we found the above approximation challenging to optimize. Specifically, to solve the MAP problem, we need to take the logarithm of all probability terms; as a result, the log-prior $\log \prod_{i} \sum_{j=1}^{k} \omega_{j} N\left(\partial L_{i} \mid 0, \varsigma_{j}\right)$ takes the form of a logarithm of a sum of exponential parts. Gradient-based optimization is the most feasible way to optimize such a log-prior [Wainwright 2006], and gradient-descent is known to be neither efficient nor stable for a complex energy function containing thousands or millions of unknowns.

In this paper, we introduce a new representation by concatenating two piece-wise continuous functions to fit the logarithmic gradient distribution:

$$
\Phi(x)=\left\{\begin{array}{ll}
-k|x| & x \leq l_{t} \\
-\left(a x^{2}+b\right) & x>l_{t}
\end{array},\right.
$$

where $x$ denotes the image gradient level and $l_{t}$ indexes the position where the two functions are concatenated. As shown in Figure 7(b), $-k|x|$, shown in green, represents the sharp peak in the distribution at the center, while $-\left(a x^{2}+b\right)$ models the heavy tails of the distribution. $\Phi(x)$ is central-symmetric, and $k, a$, and $b$ are the curve fitting parameters, which are set as $k=2.7, a=6.1 \times 10^{-4}$, and $b=5.0$.

Since we have modeled the logarithmic gradient density, the final definition of the global prior $p_{g}(L)$ is written as

$$
p_{g}(\mathbf{L}) \propto \prod_{i} e^{\Phi\left(\partial L_{i}\right)}
$$


Local prior $p_{l}(\mathbf{L})$. In this novel prior we use the blurred image to constrain the gradients of the latent image in a fashion that is very effective in suppressing ringing artifacts. This prior is motivated by the fact that motion blur can generally be considered a smooth filtering process. In a locally smooth region of the blurred image, with pixels of almost constant color (as outlined in yellow in Figure 8(a)), the corresponding unblurred image region should also be smooth (as outlined in yellow in Figure 8(b)); that is, its pixels should exhibit no salient edges. Notice that the ringing artifact, as shown in Figure 8(c), usually corresponds to patterned structures and violates this constraint.

To formulate the local prior, for each pixel $i$ in blurred image $I$, we form a local window with the same size as the blur kernel and centered at it. One example is shown in Figure 8(a) where the window is represented by the green rectangle centered at pixel $i$ highlighted by the red dot. Then, we compute the standard deviation of pixel colors in each local window. If its value is smaller than a threshold $t$, which is set to 5 in our experiments, we regard the center pixel $i$ as in region $\Omega$, i.e., $i \in \Omega$. In Figure $8(\mathrm{~d}), \Omega$ is shown as the set of all white pixels, each of which is at the center of a locally smooth window.

For all pixels in $\Omega$, we constrain the blurred image gradient to be similar to the unblurred image gradient. The errors are defined to follow a Gaussian distribution with zero mean and standard deviation $\sigma_{1}$ :

$$
p_{l}(\mathbf{L})=\prod_{i \in \Omega} N\left(\partial_{x} L_{i}-\partial_{x} I_{i} \mid 0, \sigma_{1}\right) N\left(\partial_{y} L_{i}-\partial_{y} I_{i} \mid 0, \sigma_{1}\right),
$$

where $\sigma_{1}$ is the standard deviation. The value of $\sigma_{1}$ is gradually increased over the course of optimization, as described more fully in Section 5, since this prior becomes less important as the blur kernel estimate becomes more accurate. It should be noted that although $p_{l}$ is only defined in $\Omega$, the wide footprint of the blur kernel can propagate its effects and globally suppress ringing patterns, including pixels in textured regions. In Figure 9, we show the impact of this prior. In this example, the local prior clearly helps to suppress ringing caused by errors in the blur kernel estimate.

\section{Optimization}

Our MAP problem is transformed to an energy minimization problem that minimizes the negative logarithm of the probability we have defined, i.e., the energy $E(\mathbf{L}, \mathbf{f})=-\log (p(\mathbf{L}, \mathbf{f} \mid \mathbf{I}))$. By taking all likelihood and prior definitions into (2), we get

$$
\begin{aligned}
E(\mathbf{L}, \mathbf{f}) & \propto\left(\sum_{\partial^{*} \in \Theta} w_{\kappa\left(\partial^{*}\right)}\left\|\partial^{*} \mathbf{L} \otimes \mathbf{f}-\partial^{*} \mathbf{I}\right\|_{2}^{2}\right)+ \\
& \lambda_{1}\left\|\Phi\left(\partial_{x} \mathbf{L}\right)+\Phi\left(\partial_{y} \mathbf{L}\right)\right\|_{1}+ \\
& \lambda_{2}\left(\left\|\partial_{x} \mathbf{L}-\partial_{x} \mathbf{I}\right\|_{2}^{2} \circ \mathbf{M}+\left\|\partial_{y} \mathbf{L}-\partial_{y} \mathbf{I}\right\|_{2}^{2} \circ \mathbf{M}\right)+\|\mathbf{f}\|_{1},
\end{aligned}
$$

where $\|\cdot\|_{p}$ denotes the $p$-norm operator and $\circ$ represents the element-wise multiplication operator. The four terms in (5) correspond to the terms in (2) in the same order. $M$ is a 2-D binary mask that encodes the smooth local prior $p(\mathbf{L})$ (Figure 9(e)). For any element $m_{i} \in \mathbf{M}, m_{i}=1$ if the corresponding pixel $I_{i} \in \Omega$, and $m_{i}=0$ otherwise. Here, we have parameters $w_{\kappa\left(\partial^{*}\right)}, \lambda_{1}$, and $\lambda_{2}$ derived from the probability terms:

$$
w_{\kappa\left(\partial^{*}\right)}=\frac{1}{\zeta_{\kappa\left(\partial^{*}\right)}^{2} \tau}, \quad \lambda_{1}=\frac{1}{\tau}, \quad \lambda_{2}=\frac{1}{\sigma_{1}^{2} \tau} .
$$

Among these parameters, the value of $w_{\kappa\left(\partial^{*}\right)}$ is set in the following manner. According to the likelihood definition, for any $\kappa\left(\partial^{*}\right)=q>0$, we must have $w_{\kappa\left(\partial^{*}\right)}=1 /\left(\zeta_{0}^{2} \cdot \tau \cdot 2^{q}\right)$. We

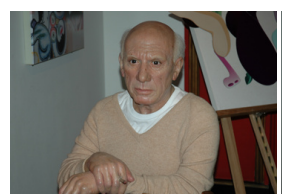

(a)

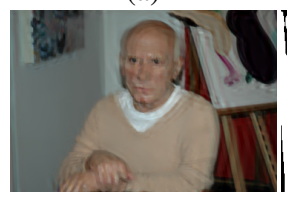

(d)

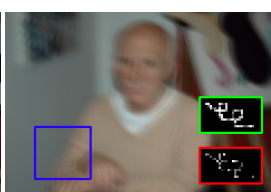

(b)

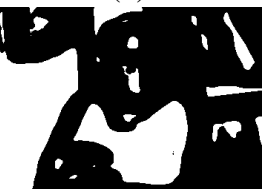

(e)

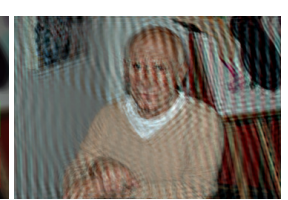

(c)
Figure 9 Effect of prior $p_{l}(\mathbf{L})$. (a) The ground truth latent image. (b) The blurred image. The ground truth blur kernel is shown in the green rectangle. We use the inaccurate kernel in the red frame to restore the blurred image to simulate one image restoration step in our algorithm. (c) The result generated from the image restoration step without using $p_{l}(\mathbf{L})$; ringing artifacts result. For comparison, we also show our image restoration results in (d) by incorporating $p_{l}(\mathbf{L})$. The computed $\Omega$ region is shown in white in (e). The ringing map is visualized in (f) by computing the color difference between (c) and (d); the map shows that $p_{l}$ is effective in supressing ringing.

usually set $1 /\left(\zeta_{0}^{2} \cdot \tau\right)=50$ in experiments. Then the value of any $w_{\kappa\left(\partial^{*}\right)}$, where $\kappa\left(\partial^{*}\right)=q>0$, can be determined as $50 /\left(2^{q}\right)$. Our configuration of $\lambda_{1}$ and $\lambda_{2}$ will be described in Section 5.3.

Directly optimizing (5) using gradient descent is slow and exhibits poor convergence since there are a large number of unknowns. In our algorithm, we optimize $E$ by iteratively estimating $\mathbf{L}$ and $\mathbf{f}$. We employ a set of advanced optimization techniques so that our algorithm can effectively handle challenging natural images.

\subsection{Optimizing $\mathrm{L}$}

In this step, we fix $\mathbf{f}$ and optimize $\mathbf{L}$. The energy $E$ can be simplified to $E_{\mathbf{L}}$ by removing constant-value terms:

$$
\begin{aligned}
E_{\mathbf{L}}= & \left(\sum_{\partial^{*} \in \Theta} w_{\kappa\left(\partial^{*}\right)}\left\|\partial^{*} \mathbf{L} \otimes \mathbf{f}-\partial^{*} \mathbf{I}\right\|_{2}^{2}\right)+\lambda_{1}\left\|\Phi\left(\partial_{x} \mathbf{L}\right)+\Phi\left(\partial_{y} \mathbf{L}\right)\right\|_{1} \\
& +\lambda_{2}\left(\left\|\partial_{x} \mathbf{L}-\partial_{x} \mathbf{I}\right\|_{2}^{2} \circ \mathbf{M}+\left\|\partial_{y} \mathbf{L}-\partial_{y} \mathbf{I}\right\|_{2}^{2} \circ \mathbf{M}\right) .
\end{aligned}
$$

While simplified, $E_{\mathbf{L}}$ is still a highly non-convex function involving thousands to millions of unknowns. To optimize it efficiently, we propose a variable substitution scheme as well as an iterative parameter re-weighting technique. The basic idea is to separate the complex convolutions from other terms in (7) so that they can be rapidly computed using Fourier transforms. Our method substitutes a set of auxiliary variables $\Psi=\left(\Psi_{x}, \Psi_{y}\right)$ for $\partial \mathbf{L}=\left(\partial_{x} \mathbf{L}, \partial_{y} \mathbf{L}\right)$, and adds extra constraints $\Psi \approx \partial \mathbf{L}$. Therefore, for each $\left(\partial_{x} L_{i}, \partial_{y} L_{i}\right) \in \partial \mathbf{L}$, there is a corresponding $\left(\psi_{i, x}, \psi_{i, y}\right) \in \Psi$. Equation (7) is therefore transformed to minimizing

$$
\begin{aligned}
E_{\mathbf{L}}= & \left(\sum_{\partial^{*} \in \Theta} w_{\kappa\left(\partial^{*}\right)}\left\|\partial^{*} \mathbf{L} \otimes \mathbf{f}-\partial^{*} \mathbf{I}\right\|_{2}^{2}\right)+\lambda_{1}\left\|\Phi\left(\Psi_{x}\right)+\Phi\left(\Psi_{y}\right)\right\|_{1} \\
& +\lambda_{2}\left(\left\|\Psi_{x}-\partial_{x} \mathbf{I}\right\|_{2}^{2} \circ \mathbf{M}+\left\|\Psi_{y}-\partial_{y} \mathbf{I}\right\|_{2}^{2} \circ \mathbf{M}\right) \\
& +\gamma\left(\left\|\Psi_{x}-\partial_{x} \mathbf{L}\right\|_{2}^{2}+\left\|\Psi_{y}-\partial_{y} \mathbf{L}\right\|_{2}^{2}\right)
\end{aligned}
$$

where $\gamma$ is a weight whose value is iteratively increased until it is sufficiently large in the final iterations of our optimization. As a result, the desired condition $\Psi=\partial \mathbf{L}$ is eventually satisfied so that minimizing $E_{\mathbf{L}}$ is equivalent to minimizing $E$. Given this variable 

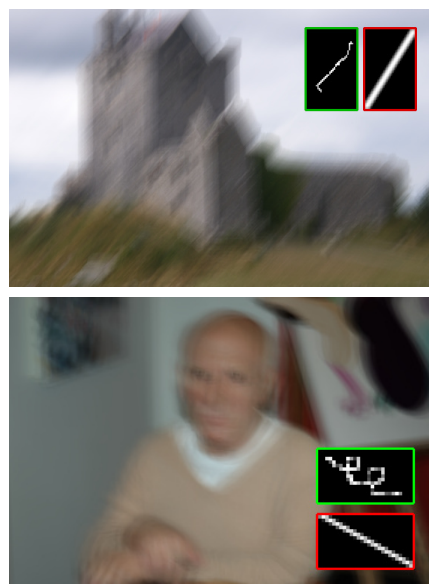

(a) Blurred image

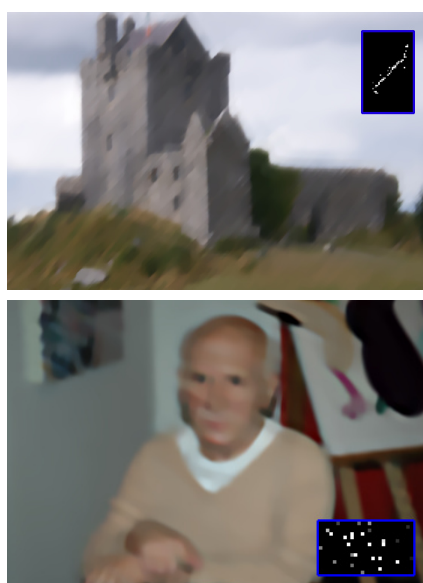

(b) Iteration 1

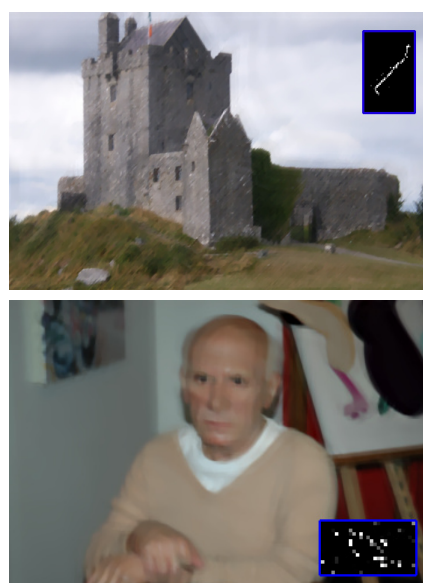

(c) Iteration 6

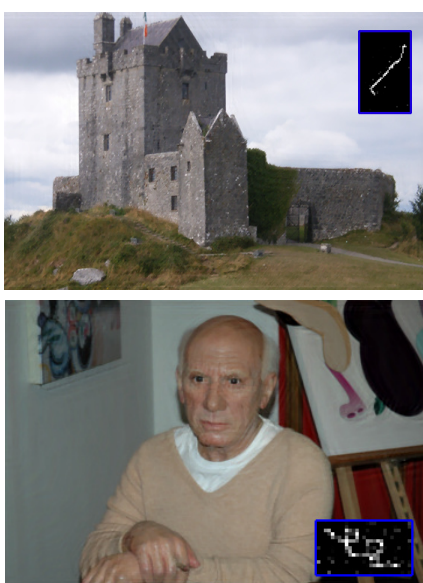

(d) Iteration 10

Figure 10 Illustration of our optimization in iterations. (a) The blurred images. The ground truth blur kernels are shown in the green rectangles. Our simple initialized kernels are shown in the red rectangles. (b)-(d) The restored images and kernels in iteration 1, 6, and 10.

substitution, we can now iterate between optimizing $\Psi$ and $\mathbf{L}$ while the other is held fixed. Our experiments show that this process is efficient and is able to converge to an optimal point, since the global optimum of $\Psi$ can be reached using a simple branching approach, while fast Fourier transforms can be used to update $\mathbf{L}$.

Updating $\Psi$. By fixing the values of $\mathbf{L}$ and $\partial^{*} \mathbf{L}$, (8) is simplified to

$$
\begin{aligned}
& E_{\Psi}^{\prime}=\lambda_{1}\left\|\Phi\left(\Psi_{x}\right)+\Phi\left(\Psi_{y}\right)\right\|_{1}+\lambda_{2}\left\|\left(\Psi_{x}-\partial_{x} \mathbf{I}\right)\right\|_{2}^{2} \circ \mathbf{M}+ \\
& \lambda_{2}\left\|\left(\Psi_{y}-\partial_{y} \mathbf{I}\right)\right\|_{2}^{2} \circ \mathbf{M}+\gamma\left\|\Psi_{x}-\partial_{x} \mathbf{L}\right\|_{2}^{2}+\gamma\left\|\Psi_{y}-\partial_{y} \mathbf{L}\right\|_{2}^{2} .(9)
\end{aligned}
$$

By simple algebraic operations to decompose $\Psi$ to all elements $\psi_{i, x}$ and $\psi_{i, y}, E_{\psi}^{\prime}$ can be further decomposed into a sum of sub-energy terms

$$
E_{\Psi}^{\prime}=\sum_{i}\left(E_{\psi_{i, x}}^{\prime}+E_{\psi_{i, y}}^{\prime}\right)
$$

where each $E_{\psi_{i, \nu}}^{\prime}, \nu \in\{x, y\}$, only contains a single variable $\psi_{i, \nu} \in \Psi_{\nu} . E_{\psi_{i, \nu}}^{\prime, \nu}$ is expressed as

$E_{\psi_{i, \nu}}^{\prime}=\lambda_{1}\left|\Phi\left(\psi_{i, \nu}\right)\right|+\lambda_{2} m_{i}\left(\psi_{i, \nu}-\partial_{\nu} I_{i}\right)^{2}+\gamma\left(\psi_{i, \nu}-\partial_{\nu} L_{i}\right)^{2}$.

Each $E_{\psi_{i, \nu}}^{\prime}$ contains only one variable $\psi_{i, \nu}$, so they can be optimized independently. Since $\Phi$ consists of four convex, differentiable pieces, each piece is minimized separately and the minimum among them is chosen. This optimization step can be completed quickly, resulting in a global minimum for $\Psi$.

Updating L. With $\Psi$ fixed, we update $\mathbf{L}$ by minimizing

$$
\begin{aligned}
E_{\mathbf{L}}^{\prime}= & \left(\sum_{\partial^{*} \in \Theta} w_{\kappa\left(\partial^{*}\right)}\left\|\partial^{*} \mathbf{L} \otimes \mathbf{f}-\partial^{*} \mathbf{I}\right\|_{2}^{2}\right)+ \\
& \gamma\left\|\Psi_{x}-\partial_{y} \mathbf{L}\right\|_{2}^{2}+\gamma\left\|\Psi_{y}-\partial_{y} \mathbf{L}\right\|_{2}^{2} .
\end{aligned}
$$

Since the major terms in $E_{\mathbf{L}}^{\prime}$ involve convolution, we operate in the frequency domain using Fourier transforms to make the computation efficient.

Denote the Fourier transform operator and its inverse as $\mathcal{F}$ and $\mathcal{F}^{-1}$ respectively. We apply the Fourier transform to all functions within the square terms in $E_{\mathbf{L}}^{\prime}$ and get

$$
\begin{gathered}
E_{\mathcal{F}(\mathbf{L})}^{\prime}=\left(\sum_{\partial^{*} \in \Theta} w_{\kappa\left(\partial^{*}\right)}\left\|\mathcal{F}(\mathbf{L}) \circ \mathcal{F}(\mathbf{f}) \circ \mathcal{F}\left(\partial^{*}\right)-\mathcal{F}(\mathbf{I}) \circ \mathcal{F}\left(\partial^{*}\right)\right\|_{2}^{2}\right) \\
\quad+\gamma\left\|\mathcal{F}\left(\Psi_{x}\right)-\mathcal{F}(\mathbf{L}) \circ \mathcal{F}\left(\partial_{x}\right)\right\|_{2}^{2}+\gamma\left\|\mathcal{F}\left(\Psi_{y}\right)-\mathcal{F}(\mathbf{L}) \circ \mathcal{F}\left(\partial_{y}\right)\right\|_{2}^{2},
\end{gathered}
$$

where $\mathcal{F}\left(\partial^{*}\right)$ is the filter in frequency domain transformed from the filter $\partial^{*}$ in image spatial domain. It can be computed by the Matlab function "psf2otf".

According to Plancherel's theorem [Bracewell 1999], which states that the sum of the square of a function equals the sum of the square of its Fourier transform, the energy equivalence $E_{\mathbf{L}}^{\prime}=E_{\mathcal{F}(\mathbf{L})}^{\prime}$ can be built for all possible values of $\mathbf{L}$. It further follows that the optimal values of variable $\mathbf{L}^{*}$ that minimize $E_{\mathbf{L}}^{\prime}$ correspond to their counterparts $\mathcal{F}\left(\mathbf{L}^{*}\right)$ in the frequency domain that minimize $E_{\mathcal{F}(\mathbf{L})}^{\prime}$ :

$$
\left.E_{\mathbf{L}}^{\prime}\right|_{\mathbf{L}^{*}}=\left.E_{\mathcal{F}(\mathbf{L})}^{\prime}\right|_{\mathcal{F}\left(\mathbf{L}^{*}\right)} .
$$

Accordingly, we compute the optimal $\mathbf{L}^{*}$ by

$$
\mathbf{L}^{*}=\mathcal{F}^{-1}\left(\arg \min _{\mathcal{F}(\mathbf{L})} E_{\mathcal{F}(\mathbf{L})}^{\prime}\right) .
$$

Since $E_{\mathcal{F}(\mathbf{L})}^{\prime}$ is a sum of quadratic energies of unknown $\mathcal{F}(\mathbf{L})$, it is a convex function and can be solved by simply setting the partial derivative $\partial E_{\mathcal{F}(\mathbf{L})}^{\prime} / \partial \mathcal{F}(\mathbf{L})$ to zero [Gamelin 2003]. The solution of $\mathbf{L}^{*}$ can be expressed as

$\mathbf{L}^{*}=\mathcal{F}^{-1}\left(\frac{\overline{\mathcal{F}(\mathbf{f})} \circ \mathcal{F}(\mathbf{I}) \circ \Delta+\gamma \overline{\mathcal{F}\left(\partial_{x}\right)} \circ \mathcal{F}\left(\Psi_{x}\right)+\gamma \overline{\mathcal{F}\left(\partial_{y}\right)} \circ \mathcal{F}\left(\Psi_{y}\right)}{\overline{\mathcal{F}(\mathbf{f})} \circ \mathcal{F}(\mathbf{f}) \circ \Delta+\gamma \overline{\mathcal{F}\left(\partial_{x}\right)} \circ \mathcal{F}\left(\partial_{x}\right)+\gamma \overline{\mathcal{F}\left(\partial_{y}\right)} \circ \mathcal{F}\left(\partial_{y}\right)}\right)$, where $\Delta=\sum_{\partial^{*} \in \Theta} w_{\kappa\left(\partial^{*}\right)} \overline{\mathcal{F}\left(\partial^{*}\right)} \circ \mathcal{F}\left(\partial^{*}\right)$ and $\overline{(\cdot)}$ represents the conjugate operator. The division is performed element-wise.

Using the above two steps we alternatively update $\Psi$ and $\mathbf{L}$ until convergence. Note that $\gamma$ in (8) controls how strongly $\Psi$ is constrained to be similar to $\partial \mathbf{L}$, and its value is set with the following consideration. If $\gamma$ is set too large, the convergence will be quite slow. On the other hand, if we fix $\gamma$ too small, the optimal solution of (8) is not the same one of (5). So we adaptively adjust the value of $\gamma$ in the optimization to keep the number of iterations small without sacrificing accuracy. In early iterations, $\gamma$ is set small ( 2 in our experiments), to stimulate significant gains for each step. Then, we double its value in each iteration so that $\Psi$ gradually approaches $\partial \mathbf{L}$. The value of $\gamma$ becomes sufficiently large at convergence. This scheme works well in our experiments, and typically uses less than 15 iterations to converge.

\subsection{Optimizing $\mathrm{f}$}

In this step, we fix $\mathbf{L}$ and compute the optimal $\mathbf{f}$. Equation (5) is simplified to 


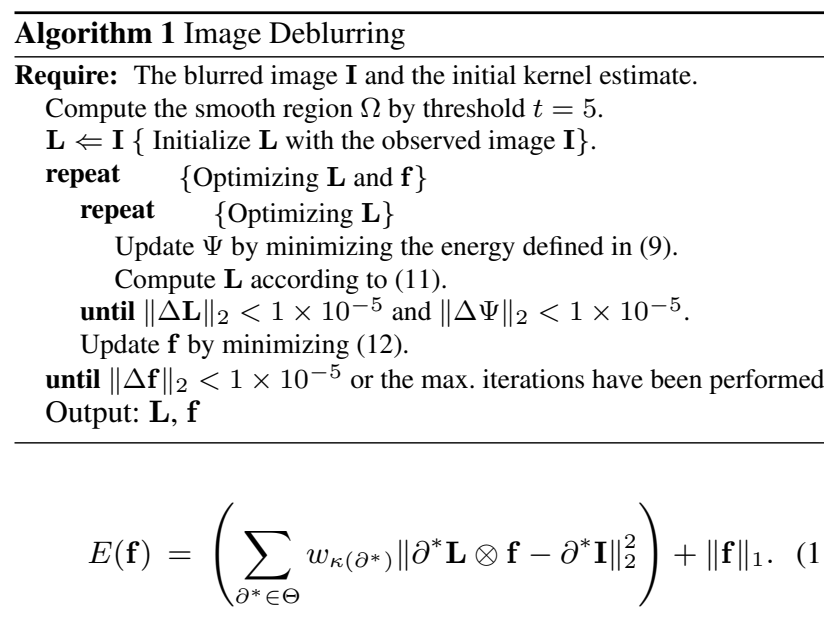

The convolution term is quadratic with respect to $\mathrm{f}$. By writing the convolution into a matrix multiplication form, similar to that in [Jia 2007; Levin et al. 2007], we get

$$
E(\mathbf{f})=\|\mathbf{A f}-\mathbf{B}\|_{2}^{2}+\|\mathbf{f}\|_{1},
$$

where $\mathbf{A}$ is a matrix computed from the convolution operator whose elements depend on the estimated latent image $\mathbf{L}$. B is a matrix determined by $\mathbf{I}$. Equation (12) is of a standard format of the problem defined in [Kim et al. 2007], and can be solved by their method which transforms the optimization to the dual problem of Equation (12) and computes the optimal solution with an interior point method. The optimization result has been shown to be very close to the global minimum.

\subsection{Optimization Details and Parameters}

We show in Algorithm 1 the skeleton of our algorithm, where the iterative optimization steps and the termination criteria are given. Our algorithm requires a rough initial kernel estimate, which can be in a form of several sparse points or simply a user-drawn line as illustrated in Figure 10.

Two parameters in our algorithm, i.e., $\lambda_{1}$ and $\lambda_{2}$ given in Equation (6), are adjustable. $\lambda_{1}$ and $\lambda_{2}$ correspond to the probability parameters in Equation (5) for the global and local priors, and their values are adapted from their initial values over iterations of the optimization. At the beginning of our blind image deconvolution the input kernel is assumed to be quite inaccurate; the two weights are therefore set to the user-given numbers (which range from 0.0020.5 and 10-25, respectively), encouraging our method to produce an initial latent image with strong edges and few ringing artifacts, as shown in Figure 10(a). Here, the fitted curve of the density of image gradients (Figure 7) contains a heavy tail, implying there exist a considerable number of large-gradient pixels in the deblurred image. This also help guide our kernel estimate in the following steps to turn aside from the trivial delta-like structure. The strongest edges are enhanced while reducing ringing artifacts. Then, after each iteration of optimization, the values of $\lambda_{1}$ and $\lambda_{2}$ are divided by $\kappa_{1}$ and $\kappa_{2}$, respectively, where we usually set $\kappa_{1} \in[1.1,1.4]$ and $\kappa_{2}=1.5$ to reduce the influence of the image prior and increase that of the image likelihood. We show in Figure 10 the intermediate results produced by our optimization. Over the iterations, the kernels are recovered and image details are enhanced.

Finally, we mention one last detail. Any algorithm that performs deconvolution in the Fourier domain must do something to avoid ringing artifacts at the image boundaries; for example, Fergus et al. [2006] process the image near the boundaries using the Matlab "edgetaper" command. We instead use the approach of Liu and Jia [2008].

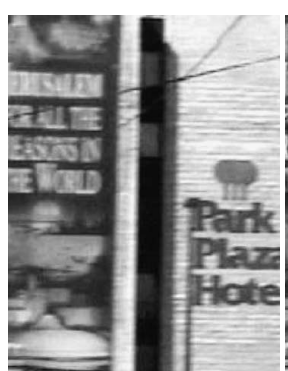

(a)

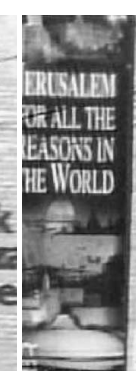

(b)

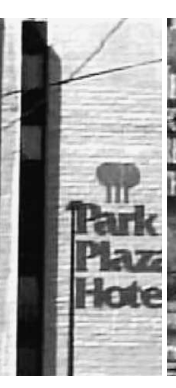

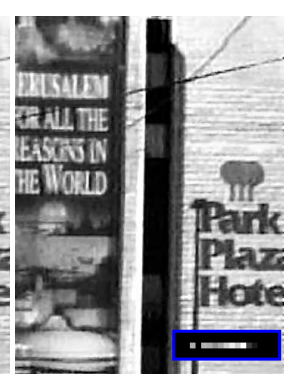

(c)
Figure 11 (a) The motion blurred image published in [Rav-Acha and Peleg 2005]. (b) Their result using information from two blurred images. (c) Our blind deconvolution result only using the blurred image shown in (a). Our estimated kernel is shown in the blue rectangle.

\section{Experimental Results}

Our algorithm contains novel approaches to both blur kernel estimation and image restoration (non-blind deconvolution). To show the effectiveness of our algorithm in both of these steps as well as a whole, we begin with a non-blind deconvolution example in Figure 12. The captured blurred image (a) contains CCD camera noise. The blur PSF is recorded as the blur of a highlight, which is further refined by our kernel estimation, as shown in (e). This kernel is used as the input of various image restoration methods to deconvolve the blurred image. We compare our result against those of the Richardson-Lucy method and the method of Levin et al. [2007] (we use code from the authors and select the result that looks best to us after tuning the parameters). Our results exhibits sharper image detail (e.g., the text in the close-ups) and fewer artifacts (e.g., the ringing around book edges) than the alternatives.

We next show a blind deconvolution example in Figure 13, consisting of two image examples captured with a hand-held camera; the blur is from camera shake, and the ground truth PSF is unknown. In particular, the image example shown in the second row is blurred by a large-size kernel, which is challenging for kernel estimation. We compare our restored image results to those produced by two other state-of-the-art algorithms. For the result of Fergus et al. [2006], we used code from the authors and hand-tuned parameters to produce the best possible results. For the result of Jia [2007] we selected patches to minimize alpha matte estimating error. In comparison, our results exhibit richer and clearer structure.

To demonstrate the effectiveness of our technique, we also compare against the results of other methods that utilize additional input. Rav-Acha and Peleg et al. [2005] use two blurred images with different camera motions to create the result in Figure 11. Surprisingly, our output computed from only one blurred image is comparable. Yuan et al. [2007] use information from two images, one blurry one noisy, to create the result in Figure 14. In comparison, our result from just the single blurry image does not contain the same amount of image details, but is still of high quality due to our accurate kernel reconstruction. Finally, Ben-Ezra and Nayar [2004] acquire a blur kernel using a video camera that is attached to a still camera, and then use the kernel to deconvolve the blurred photo produced by the still camera. Their result is shown in Figure 15. Our algorithm produces a deblurred result with a similar level of sharpness.

Finally, three more challenging real examples are shown in Figure 16, all containing complex structures and blur from a variety of camera motions. The ringings, even round strong edges and textures, are significantly reduced. The remaining artifact is caused mainly by the fact that the motion blur is not absolutely spatiallyinvariant. Using a hand-held camera, slight camera rotation and motion parallax are easily introduced [Shan et al. 2007]. 


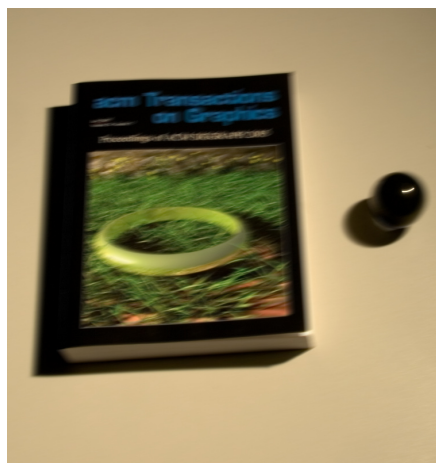

(a) Blurred image

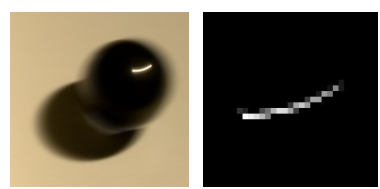

(b)

\begin{tabular}{|l|l|}
\hline Methods & $\begin{array}{l}\text { Running } \\
\text { time (s) }\end{array}$ \\
\hline RL & 54.90 \\
Levin et al. & 477.56 \\
Ours & 38.48 \\
\hline
\end{tabular}

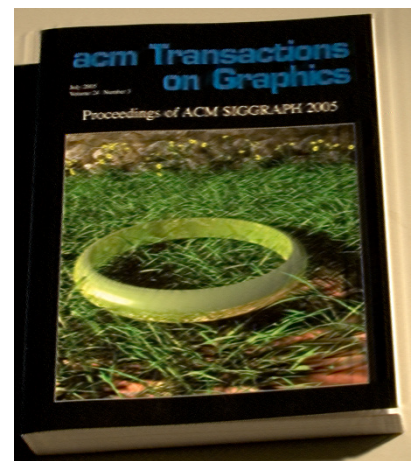

(c) Richardson-Lucy
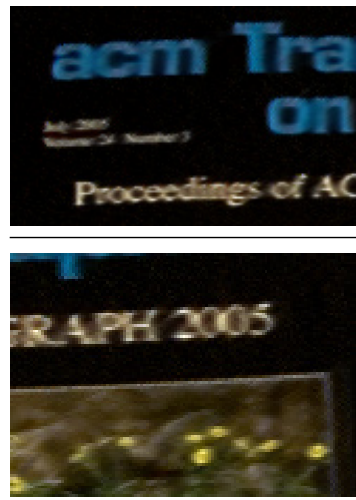

(f)

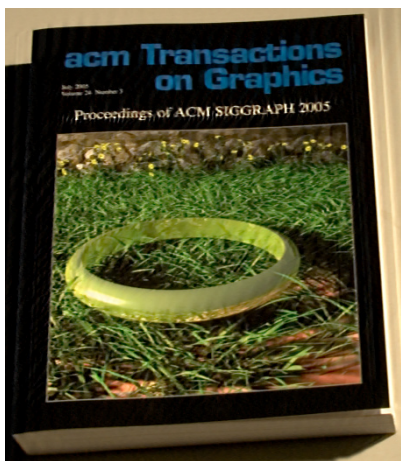

(d) [Levin et al. 2007]
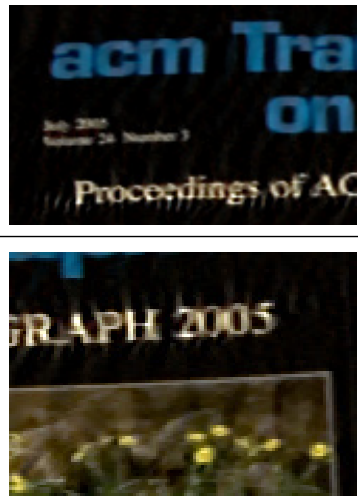

(g)

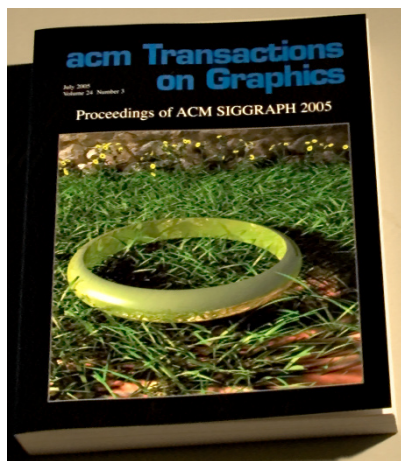

(e) Our result
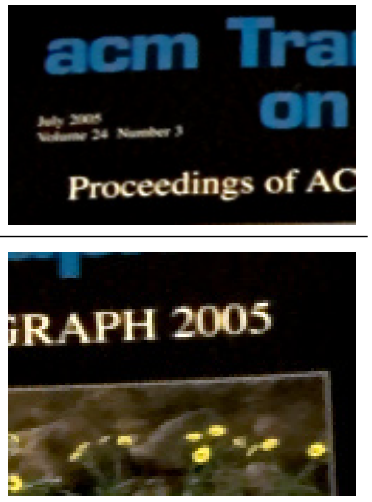

(h)

Figure 12 Non-blind deconvolution. (a) The captured blurry photograph contains a SIGGRAPH proceeding and a sphere for recording the blur PSF. The recovered kernel is shown in (b). Using this kernel, the image restoration methods deconvolve the blurred image and we show in (c) the result of RL algorithm and (d) the result of the sparse prior method [Levin et al. 2007]. Our non-deconvolution result is shown in (e) also using the kernel (b). (f)-(h) Close-ups extracted from (b)-(d), respectively. The running times of different algorithms are also shown.

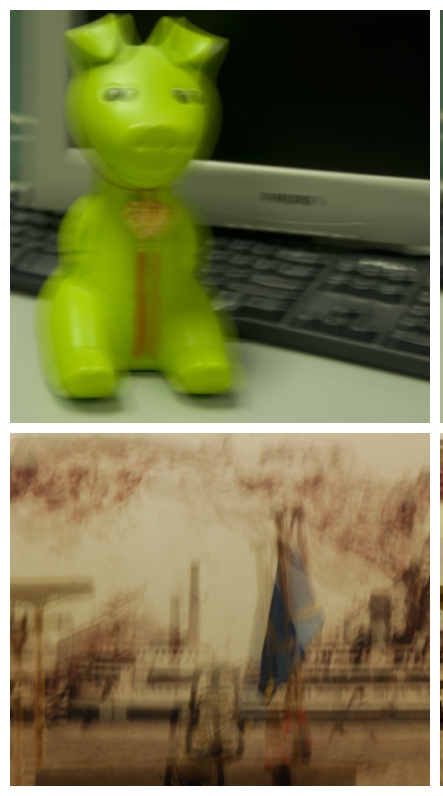

(a) Blurred images

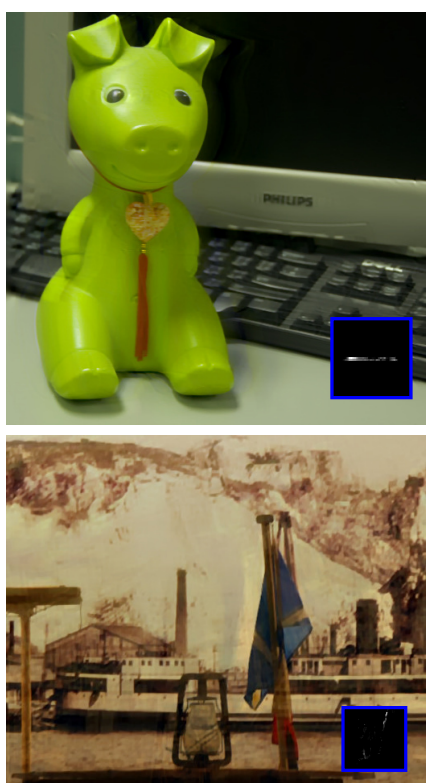

(b) Our results

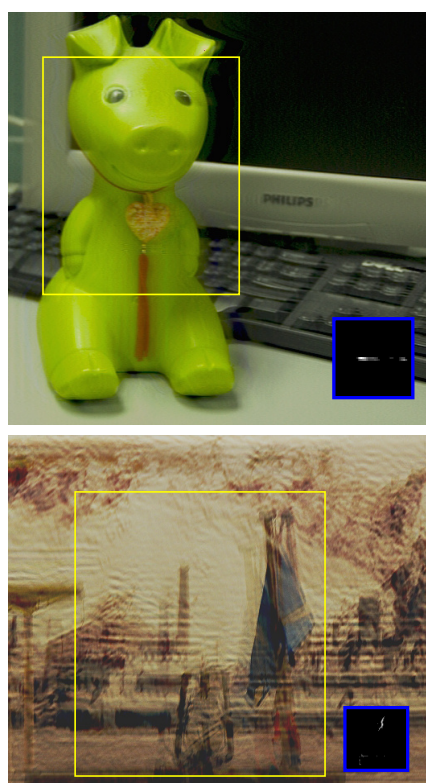

(c) [Fergus et al. 2006]

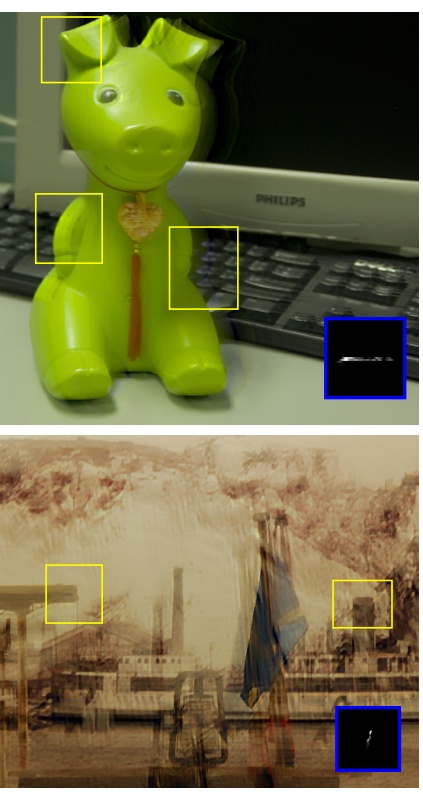

(d) [Jia 2007]

Figure 13 Blind deconvolution. (a) Our captured blur images. (b) Our results (by estimating both kernels and latent images). The deblurring results of (c) Fergus et al. [2006] and (d) Jia [2007]. The yellow rectangles indicate the selected patches for estimating kernels in (c) and the windows for computing alpha mattes and estimating kernels in (d) respectively. Both Fergus et al. [2006] and Jia [2007] use RL deconvolution to restore the blurred image. The estimated kernels are shown in the blue rectangles. 

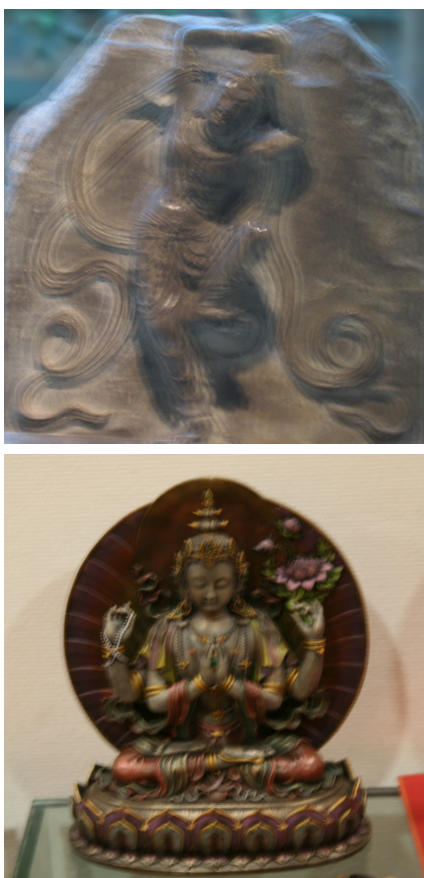

(a) Blurred images
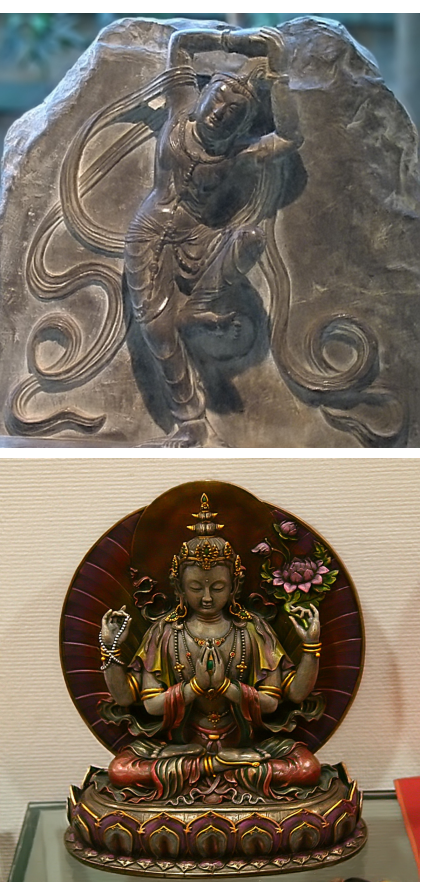

(b) [Yuan et al. 2007]
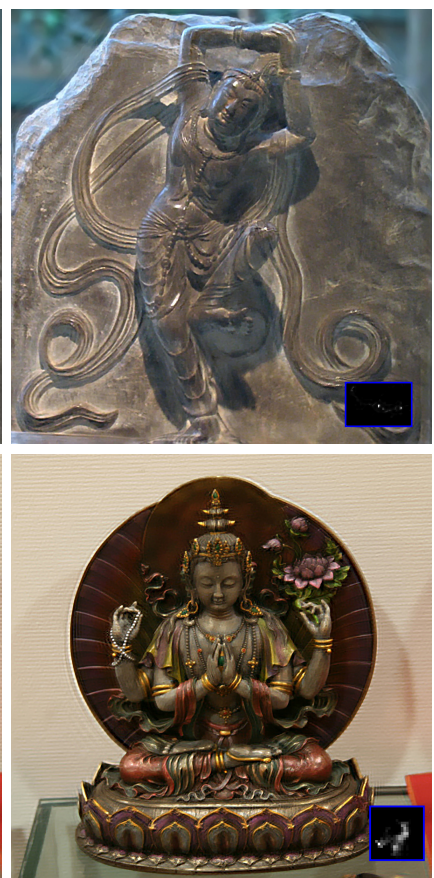

(c) Our results
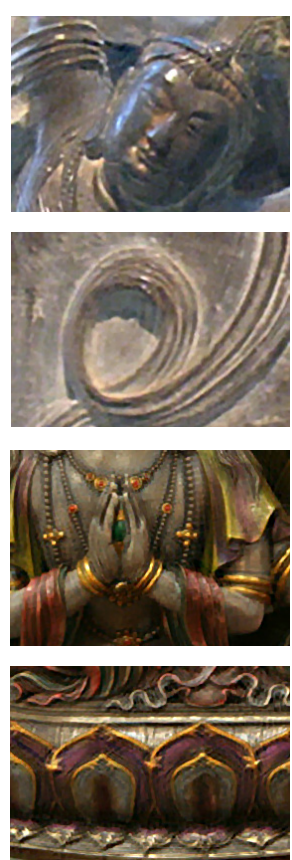

(d)

Figure 14 Statuary example. (a) The input blurred images published in [Yuan et al. 2007]. (b) Their results using a pair of images, one blurry and one noisy. (c) Our blind deconvolution results from the blurred images only. (d) Some close-ups of our results.

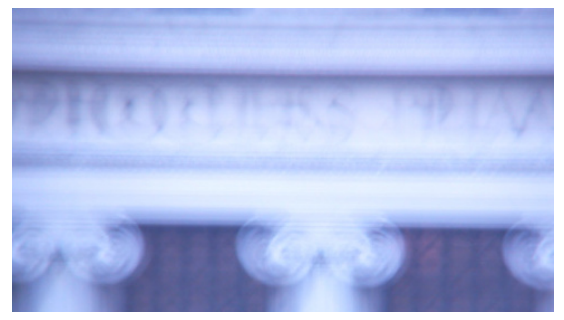

(a) Blurred image

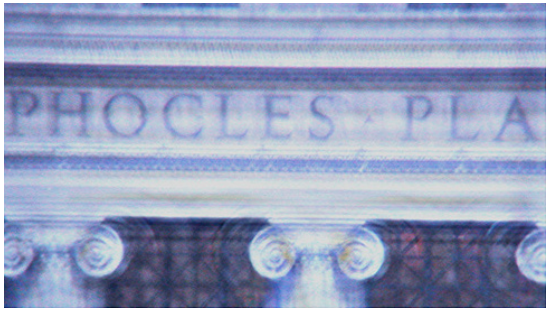

(b) [Ben-Ezra and Nayar 2004]

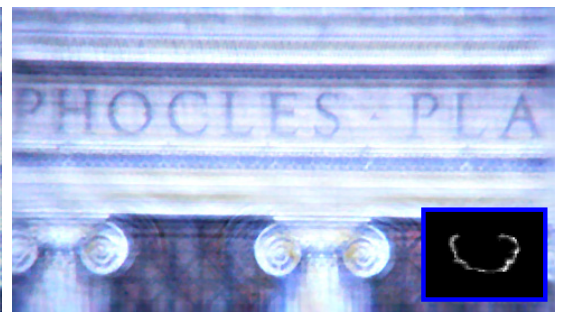

(c) Our result

Figure 15 (a) A motion blurred image of a building from the paper of Ben-Ezra and Nayar [2004]. (b) Their result using information from an attached video camera to estimate camera motion. (c) Our blind deconvolution result from the single blurred image only.

\section{Conclusion and Discussion}

In this paper, we have proposed a novel image deconvolution method to remove camera motion blur from a single image by minimizing errors caused by inaccurate blur kernel estimation and image noise. We introduced a unified framework to solve both nonblind and blind deconvolution problems. Our main contributions are an effective model for image noise that accounts for its spatial distribution, and a local prior to suppress ringing artifacts. These two models interact with each other to improve unblurred image estimation even with a very simple and inaccurate initial kernel after our advanced optimization process is applied.

Previous techniques et al. [Fergus et al. 2006] have avoided computing an MAP solution because trivial solutions, such a delta kernel (which provides no deblurring), can represent strong local minima. The success of our MAP approach is principally due to our optimization scheme that re-weights the relative strength of priors and likelihoods over the course of the optimization. At the beginning of our optimization the kernel is likely erroneous, so we do not emphasize the likelihood (data-fitting) term. Instead, the global prior is emphasized to encourage the reconstruction of strong edges according to the gradient magnitude distribution. We also emphasize the local prior in order to suppress ringing artifacts which might induce incorrect image structures and confuse the kernel estimation. Then, once strong edges are reconstructed, we gradually increase the likelihood term in later iterations in order to fine-tune the kernel and recover details in the latent image. We have found that this re-weighting approach can avoid the delta kernel solution even if it is used as the initialization of the kernel.

We have found that our technique can successfully deblur most motion blurred images. However, one failure mode occurs when the blurred image is affected by blur that is not shift-invariant, e.g., from slight camera rotation or non-uniform object motion. An interesting direction of future work is to explore the removal of nonshift-invariant blur using a general kernel assumption.

Another interesting observation that arises from our work is that blurred images contain more information than we usually expect. Our results show that for moderately blurred images, edge, color and texture information can be satisfactorily recovered. A successful motion deblurring method, thus, makes it possible to take advantage of information that is currently buried in blurred images, which may find applications in many imaging-related tasks, such as image understanding, 3D reconstruction, and video editing. 

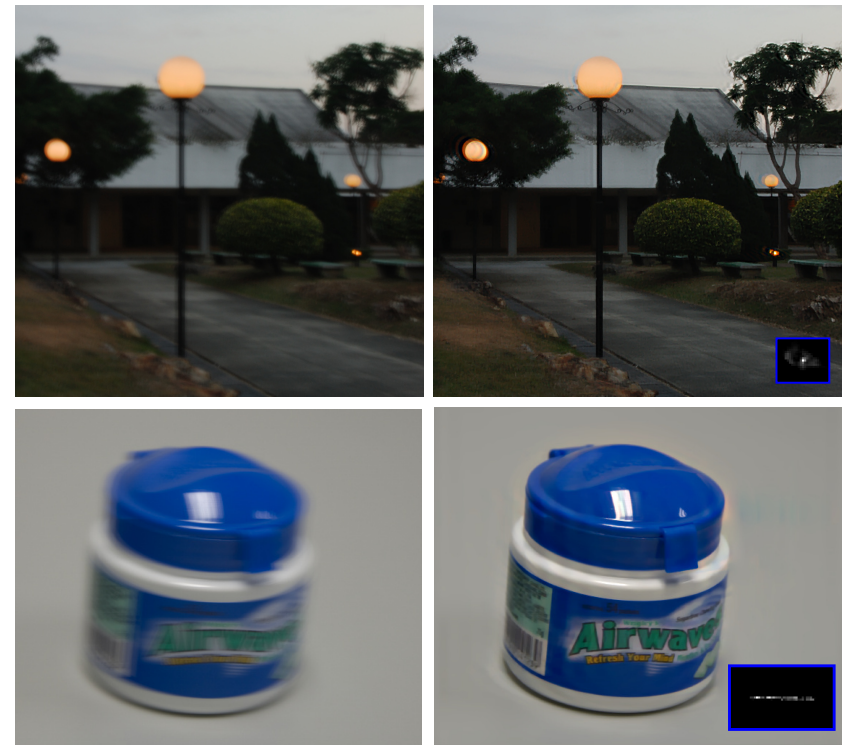

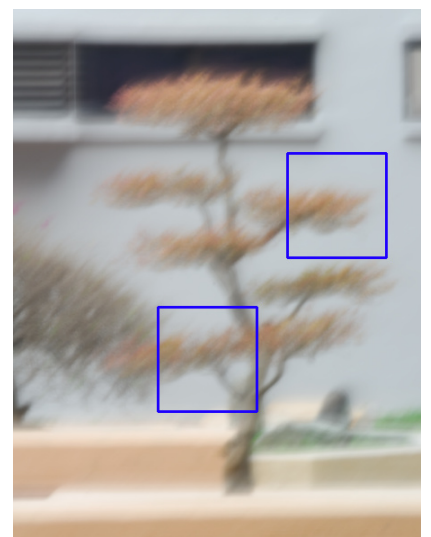

(a)

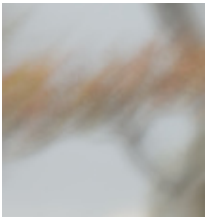

(c)

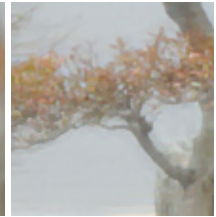

(d)

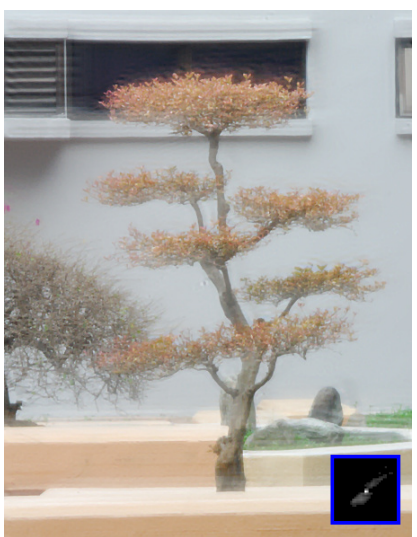

(b)

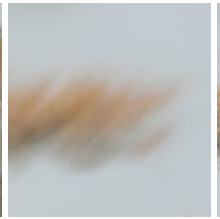

(e)

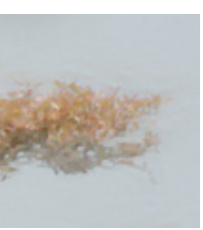

(f)
Figure 16 More results. (a) The captured blur images. (b) Our results. (c)-(f) Close-ups of blurred/unblurred image regions extracted from the last example.

\section{Acknowledgements}

We thank all reviewers for their constructive comments. This work was supported by a grant from the Research Grants Council of the Hong Kong Special Administrative Region, China (Project No. 412307).

\section{References}

Ben-EzRA, M., AND NAYAR, S. K. 2004. Motion-based motion deblurring. TPAMI 26, 6, 689-698.

Bracewell, R. N. 1999. The Fourier Transform and Its Applications. McGraw-Hill.
Donatelli, M., Estatico, C., Martinelli, A., And SerraCAPIZZANO, S. 2006. Improved image deblurring with antireflective boundary conditions and re-blurring. Inverse Problems 22, 6, 2035-2053.

Fergus, R., Singh, B., Hertzmann, A., Roweis, S. T., And FREEMAN, W. 2006. Removing camera shake from a single photograph. ACM Transactions on Graphics 25, 787-794.

Gamelin, T. W. 2003. Complex Analysis. Springer.

JIA, J. 2007. Single image motion deblurring using transparency. In $C V P R$.

KIM, S. K., AND PAIK, J. K. 1998. Out-of-focus blur estimation and restoration for digital auto-focusing system. Electronics Letters 34, 12, 1217 - 1219.

Kim, S.-J., KoH, K., Lustig, M., And Boyd, S. 2007. An efficient method for compressed sensing. In ICIP.

Levin, A., Fergus, R., Durand, F., And Freeman, B. 2007. Image and depth from a conventional camera with a coded aperture. In SIGGRAPH.

LiKas, A., AND Galatsanos, N. 2004. A Variational Approach for Bayesian Blind Image Deconvolution. IEEE Transactions on Signal Processing 52, 8, 2222-2233.

LIU, R., AND JIA, J. 2008. Reducing boundary artifacts in image deconvolution. In ICIP.

LUCY, L. 1974. Bayesian-based iterative method of image restoration. Journal of Astronomy 79, 745-754.

Miskin, J., AND MACKAY, D. 2000. Ensemble learning for blind image separation and deconvolution. Advances in Independent Component Analysis, 123-141.

Neelamani, R., Choi, H., And Baraniuk, R. G. 2004. ForWaRD: Fourier-wavelet regularized deconvolution for illconditioned systems. IEEE Transactions on Signal Processing $52,418-433$.

Raskar, R., Agrawal, A., And Tumblin, J. 2006. Coded exposure photography: Motion deblurring using fluttered shutter. ACM Transactions on Graphics 25, 3, 795-804.

RaV-ACHA, A., AND Peleg, S. 2005. Two motion blurred images are better than one. Pattern Recognition Letters 26, 311-317.

Roth, S., AND BLACK, M. J. 2005. Fields of experts: A framework for learning image priors. In $C V P R$.

Shan, Q., XiOng, W., AND Jia, J. 2007. Rotational motion deblurring of a rigid object from a single image. In ICCV.

Simon, M. K. 2002. Probability Distributions Involving Gaussian Random Variables: A Handbook for Engineers, Scientists and Mathematicians. Springer.

WAINWRIGHT, M. J. 2006. Estimating the "wrong" graphical model: Benefits in the computation-limited setting. Journal of Machine Learning Research, 1829-1859.

Weiss, Y., AND FreEman, W. T. 2007. What makes a good model of natural images? In $C V P R$.

WIENER, N. 1964. Extrapolation, Interpolation, and Smoothing of Stationary Time Series. MIT Press.

YuAN, L., Sun, J., QUAN, L., AND Shum, H.-Y. 2007. Image Deblurring with Blurred/Noisy Image Pairs. In SIGGRAPH. 